„WIR WAREN UNS EINIG, DASS DAS BIOSPEKTRUM FÜR ANDERE

MOLEKULAR ORIENTIERTE BIOLOGISCHE FACHGESELLSCHAFTEN

OFFEN BLEIBEN SOLLTE, UM DER ZERSPLITTERUNG DER BIOLOG/INN/EN

UND BIOCHEMIKER/INNEN ENTGEGENZUWIRKEN UND MIT EINER

STIMME SPRECHEN ZU KÖNNEN.“

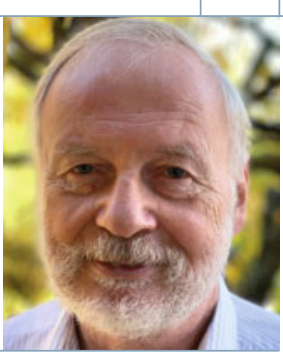

Hartmut Michel

\title{
25 Jahre BIOspektrum: mit Meilensteinen der Forschung in die Zukunft
}

DOI: $10.1007 / \mathrm{s} 12268-020-1503-2$ (C) Der Autor 2020

Wie schnell doch die Zeit vergeht! 25 Jahre sind jetzt schon seit dem Erscheinen der ersten Ausgabe unseres BIOspektrums vergangen. Ein kurzer Blick zurück: Das BIOspektrum wurde als neue Mitgliederzeitschrift der Gesellschaft für Biochemie und Molekularbiologe (GBM, damals noch Gesellschaft für Biologische Chemie, GBCh) und der Vereinigung für Allgemeine und Angewandte Mikrobiologie (VAAM) gegründet. Die Motive waren von Seiten der GBM, dass es notwendig wurde, Prof. Dr. Lothar Jaenicke und Dr. Klaus Beaucamp zu entlasten, die zuständig für den redaktionellen Teil der Grünen Blätter waren, dem damaligen, dreimal pro Jahr produzierten Mitteilungsblatt der GBM. Gleichzeitig sollten die GBM-Mitglieder häufiger, aktueller und kostengünstiger informiert werden. Die VAAM konnte Teile ihrer eigenen, professionelleren Vorläuferzeitschriften Forum Mikrobiologie und Bioengineering mitsamt einem wesentlichen Teil des Redaktionsteams einbringen. Der damalige VAAM-Präsident, der viel zu früh verstorbene Prof. Dr. Achim Kröger, und ich waren uns einig, dass das BIOspektrum für andere molekular orientierte biologische Fachgesellschaften offen bleiben sollte, um der Zersplitterung der molekular orientierten Biologen und Biochemiker entgegenzuwirken und mit einer Stimme sprechen zu können. Für die Ausbildung der Studierenden sollten auf Deutsch geschriebene kurze Übersichtsartikel, Schlaglichtartikel zu aktuellen Themen, methodisch orientierte Arbeiten und aktuelle Nachrichten das BIOspektrum prägen. Die Gesellschaft für Genetik (GfG) und die Deutsche Gesellschaft für Experimentelle und Klinische Pharmakologie und Toxikologie (DGPT) nutzen jetzt ebenfalls das BIOspektrum. Mit VBIO, dem Verband Biologie, Biowissenschaften \& Bionmedizin, wurde eine Kooperation initiiert. Dadurch wurde das BIOspektrum aktueller und thematisch vielfältiger.

Was ist seit der ersten BIOspektrum-Ausgabe sonst noch geschehen? Politisch ist die Welt unsicherer geworden, der Populismus hat zugenommen, in vielen Ländern ist die Freiheit der Wissenschaft in Gefahr. Dem müssen auch wir Biologen und Biochemiker entgegentreten! In den Biowissenschaften werden neue bahnbrechende Ergebnisse mit einem Tempo veröffentlicht, das ich nicht für möglich gehalten hätte. Ein Beispiel: Nach der Kristallisation und Strukturaufklärung des bakteriellen photo- synthetischen Reaktionszentrums hatte ich geglaubt, noch nach meiner Emeritierung an der Kristallisation und Strukturaufklärung des pflanzlichen Photosystem II arbeiten zu können. Weit gefehlt! Und die Geschwindigkeit des Schaffens neuen Wissens in den Biowissenschaften nimmt immer weiter zu! Dazu hat in erster Linie die Entwicklung neuer Methoden beigetragen. In der optischen Mikroskopie wurde mithilfe von Fluoreszenztechniken die Auflösungsgrenze immer weiter nach unten verschoben. Der größte Durchbruch hat sich wohl in der biologischen Strukturforschung ergeben. Die resolution revolution der Elektronenmikroskopie (single particle electron cryomicroscopy) macht heute sogar tatsächlich atomare Auflösungen von 1,2 A möglich (Yip KM et al., Nature (2020), https://doi. org/10.1038/s41586-020-2833-4 und Nakane T et al., bioRxiv, https://www.biorxiv.org/content /10.1101/2020.05.22.110189v1). Die Vorteile der Elektronenmikroskopie sind, verglichen mit der Röntgenkristallographie, der wesentlich geringere Materialbedarf und die Umgehung der zeitaufwändigen Kristallisation, die sich zudem häufig als unmöglich erweist. Dadurch kann in der biologischen Strukturforschung auf die Verwendung teurer Synchrotrone und freier Elektronenlaser (XFEL) weitgehend verzichtet werden. Genome editing, insbesondere mithilfe von CRISPR-Cas, hat die Biotechnologie revolutioniert. In der Arzneimittelentwicklung und Therapie diverser Erkrankungen haben monoklonale Antikörper einen festen Platz erobert, wobei ich allerdings der Meinung bin, dass klassische niedermolekulare Pharmaka vorzuziehen sind. Leider ist ihre Entwicklung schwieriger und teurer, der Einsatz dafür ohne Kühlkette möglich und die Herstellung kostengünstiger. Die Methoden der Genomsequenzierung haben sich ebenfalls revolutionär entwickelt: Humangenome können in hoher Qualität und mit erschwinglichem Preis in nur wenigen Tagen entschlüsselt werden. Ganz besonders fasziniert mich die Sequenzierung alter Genome in der Archäogenetik, die eindeutige Schlüsse auf die Besiedlung der Welt durch den modernen Menschen out of Africa zulässt. Wer hätte gedacht, dass wir Europäer das Ergebnis der Vermischung der Genome dreier Menschengruppen des Homo sapiens sind? Wer hätte geglaubt, dass auch der Neandertaler einer unser direkten Vorfahren ist, von dem wir rund zwei Prozent unserer Gene haben?

Was wird die Zukunft bringen? Was sind die Herausforderungen für die Zukunft? Ziel der Bio- medizin muss es sein, den menschlichen Körper so gut wie möglich zu verstehen. Unser Verständnis muss bei der Verschmelzung von Samen- und Eizelle beginnen. Man muss wissen, welche Proteine in welchem Zelltyp vorhanden und aktiv sind, und wo sie lokalisiert sind. Die Zahl der identifizierten Zelltypen nimmt ständig zu. Man muss wissen, was die Funktion eines jeden Proteins des menschlichen Körpers ist und wie es arbeitet. Single cell proteomics scheint Realität zu werden. Man muss wissen, was bei den Zellteilungen passiert, welche Gene und Proteine aktiviert oder deaktiviert werden, wie Gewebe und Organe gebildet werden.

Es gibt viele spannende Fragen, die auf eine Antwort warten: Was ist die tatsächliche Ursache der Alzheimerschen Erkrankung und anderer neurologischer Pathologien? Warum und wie altern wir? Warum müssen wir schlafen und was genau passiert im Schlaf? Die allergrößte Herausforderung stellt allerdings das Verständnis unseres Gehirns und seiner höheren Funktionen dar.

Wenn ich heute mit der Wissenschaft beginnen würde, wäre die Altersforschung mein Favorit. Dem BIOspektrum wünsche ich allerdings, dass es nicht altert und jung und attraktiv bleibt.

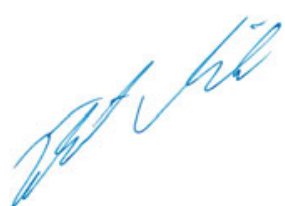

Hartmut Michel

Direktor am Max-Planck-Institut für Biophysik und apl. Professor an der Universität Frankfurt a. M.

Funding note: Open Access funding enabled and organized by Projekt DEAL. Open Access: Dieser Artikel wird unter der Creative Commons Namensnennun 4.0 International Lizenzveröffentlicht, welche die Nutzung, Vervielfältigung Bearbeitung Verbreitung und Wiedergabe in jeglichem Medium und Format erlaubt, sofern Sie den/die ursprünglichen Autor(en) und die Quelle ordnungsgemäß nennen, einen Link zur Creative Commons Lizenz beifügen und angeben, ob Änderungen vorgenommen wurden. Die in diesem Artikel enthaltenen Bilder und sonstiges Drittmaterial unterliegen ebenfalls der genannten Creative Commons Lizenz, sofern sich aus der Abbildungslegende nichts anderes ergibt. Sofern das betreffende Material nicht unter der genannten Creative Commons Lizenz steht und die betreffende Handlung nich Weiterverwendun Rechteinhabers einzuholen. Weitere Details zur Lizenz entnehmen Sie bitte der Lizenzinformation auf http://creativecommons.org/licenses/by/4.0/deed.de.

\section{Korrespondenzadresse:}

Prof. Dr. Dr. h.c. Hartmut Michel

Max-Planck-Institut für Biophysik

Max-von-Laue-Straße 3

D-60438 Frankfurt a. M.

hartmut.michel@biophys.mpg.de 